\title{
Fluo-Fluo translation based on deep learning
}

\author{
Zhengfen Jiang (姜正芬) $)^{1}$, Boyi Li (李博艺) ${ }^{2}$, Tho N. H. T. Tran (陈 诗 $)^{2}$, Jiehui Jiang (蒋皆恢) ${ }^{1}$, Xin Liu (刘 欣 $)^{2,3^{*}}$ ， and \\ Dean Ta (他得安) $)^{2,4^{* *}}$ \\ ${ }^{1}$ School of Communication \& Information Engineering, Shanghai University, Shanghai 200444, China \\ ${ }^{2}$ Academy for Engineering \& Technology, Fudan University, Shanghai 200433, China \\ ${ }^{3}$ State Key Laboratory of Medical Neurobiology, Fudan University, Shanghai 200433, China \\ ${ }^{4}$ Center for Biomedical Engineering, Fudan University, Shanghai 200433, China \\ *Corresponding author: xinliu.c@gmail.com \\ ${ }^{*}$ Corresponding author: tda@fudan.edu.cn \\ Received November 11, 2021 | Accepted December 13, २०२1 | Posted Online January 4, 2022
}

\begin{abstract}
Fluorescence microscopy technology uses fluorescent dyes to provide highly specific visualization of cell components, which plays an important role in understanding the subcellular structure. However, fluorescence microscopy has some limitations such as the risk of non-specific cross labeling in multi-labeled fluorescent staining and limited number of fluorescence labels due to spectral overlap. This paper proposes a deep learning-based fluorescence to fluorescence (FluoFluo) translation method, which uses a conditional generative adversarial network to predict a fluorescence image from another fluorescence image and further realizes the multi-label fluorescent staining. The cell types used include human motor neurons, human breast cancer cells, rat cortical neurons, and rat cardiomyocytes. The effectiveness of the method is verified by successfully generating virtual fluorescence images highly similar to the true fluorescence images. This study shows that a deep neural network can implement Fluo-Fluo translation and describe the localization relationship between subcellular structures labeled with different fluorescent markers. The proposed Fluo-Fluo method can avoid non-specific cross labeling in multi-label fluorescence staining and is free from spectral overlaps. In theory, an unlimited number of fluorescence images can be predicted from a single fluorescence image to characterize cells.
\end{abstract}

Keywords: deep learning; conditional generative adversarial network; fluorescence image; image translation. DOI: 10.3788/COL202220.031701

\section{Introduction}

Fluorescence microscopy uses fluorescent dyes to provide highly specific visualization of cell components, which plays an important role in understanding the subcellular structure of cells ${ }^{[1]}$. However, challenges remain in fluorescence microscopy. Firstly, the labeling of multiple fluorescent molecules on the antibody may have the risk of non-specific cross labeling and affect the immune activity of the antibody ${ }^{[2]}$. Secondly, when there are many different fluorescence images in the sample, the number of distinguishable fluorescent colors in the visible light range is usually limited to four due to the limitation of overlapping emission spectra of fluorescent dyes ${ }^{[3]}$.

Recently, convolutional neural networks (CNNs) have been proved to have unprecedented performance in microscopy image analysis tasks ${ }^{[4-9]}$. In 2018, Christiansen et al. ${ }^{[10]}$ used CNNs to generate virtual fluorescence images from the unlabeled transmitted light images to identify the location and texture of nuclei and membranes, the health of cells, the types of cells, and the subcellular structures. In 2020, Nguyen et al. ${ }^{[11]}$ predicted vinculin from $4^{\prime}$,6-diamidino-2-phenylindole (DAPI)/Hoechst and F-actin. They demonstrated that the feasibility of a deep learning method in predicting the fluorescence images depends on the correlation between input images and output images. Furthermore, in 2021, Shigene et al. ${ }^{[12]}$ successfully predicted cytoskeletal proteins from actin, further proving that CNNs can predict the location of functionally related proteins.

The conditional generative adversarial network (cGAN) $)^{[13]}$ is an effective deep learning model, which is composed of a generator and a discriminator. Through competition between the generator and the discriminator, cGAN can generate high-quality images ${ }^{[14,15]}$. In this paper, we propose a new fluorescence to fluorescence (Fluo-Fluo) translation method, which is achieved by cGAN. In theory, an unlimited number of fluorescence images can be predicted from one fluorescence image by cGAN. Furthermore, cGAN can also realize virtual multi-label fluorescence staining that can avoid non-specific cross labeling and spectral overlaps in multi-label fluorescence staining 


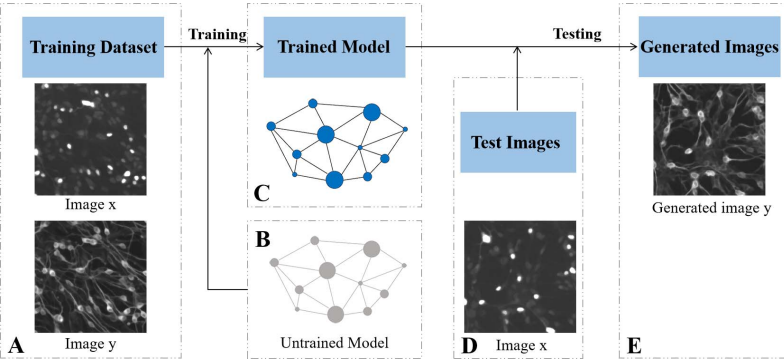

Fig. 1. System for Fluo-Fluo translation based on CGAN. (A) The training dataset is composed of fluorescence images $x$ and $y$ in the same field of view. (B) A deep neural network is composed of untrained parameters. (C) The deep neural network trained with data in (A). (D) Test image. (E) Based on the trained deep learning model, the fluorescence image $y$ is predicted from the fluorescence image $x$.

experiments. To our knowledge, the application of cGAN in Fluo-Fluo translation is not reported previously.

Figure 1 illustrates the cGAN-based system for Fluo-Fluo translation. Briefly, in this work, cGAN is employed to predict the location of III-tubulin (TuJ1) protein, from Islet1 protein to predict the location and intensity of membrane from DAPI, to predict whether the cell dies from Hoechst, to predict microtubule-associated protein-2 (MAP2) and neurofilament-F $(\mathrm{NFH})$ from DAPI, and to further realize multi-label fluorescent staining. Especially to improve the network output quality, a new loss function combining the advantages of the L1 loss function and the multi-scale structural similarity (MS-SSIM) loss function $^{[16]}$ is constructed.

\section{Principle}

\subsection{Conditional generative adversarial network}

The purpose of image-to-image translation is to learn the mappings between different domains in order to complete the image translation across domains ${ }^{[17]}$. To predict a fluorescence image from another fluorescence image, we adopt the cGAN-driven architecture Pix $2 \mathrm{Pix}^{[13]}$. The cGAN training process involves two different networks, namely the generator network and the discriminator network. The generator network attempts to generate an output image highly similar to the ground truth, and the discriminator network tries to distinguish between the ground truth and the image output by the generator. Finally, the trained network can predict a fluorescence image from another fluorescence image. The cGAN framework for Fluo-Fluo translation based on deep learning is demonstrated in Fig. 2.

\subsection{Loss function}

The selection of loss function is a key component of the deep neural network design. The L1 loss function can keep the brightness and color unchanged, but it assumes that the influence of noise and the local characteristics of the image are independent. The MS-SSIM loss function is an index that integrates human

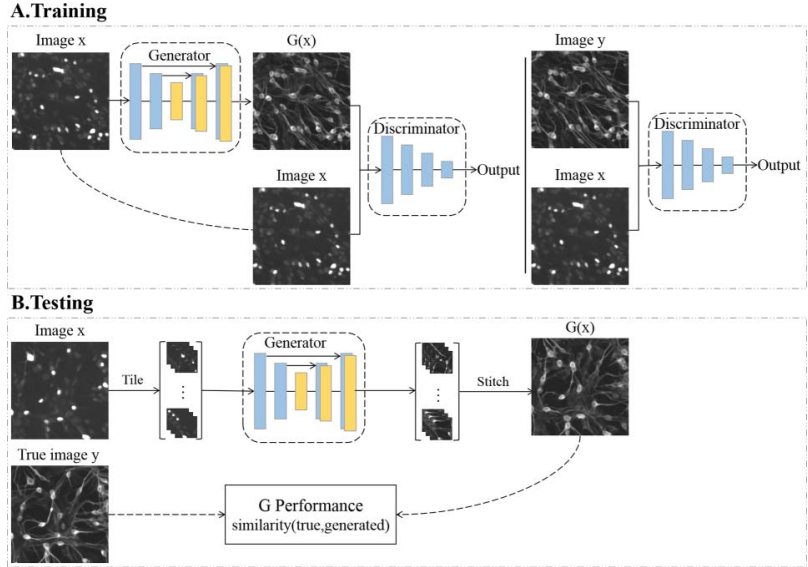

Fig. 2. cGAN framework for Fluo-Fluo translation based on deep learning. (A) The generator network attempts to generate image $y$ with respect to image $x$, and the discriminator network attempts to distinguish between the generated image $y$ and the true image $y$. There is a competitive relationship between these two networks. Briefly, image $x$ is used as the input of the generator to obtain the generated image $G(x)$, and then $G(x)$ and $x$ are combined as the input of the discriminator. During training, two error functions are calculated: (i) L1 and MS-SSIM loss functions are used to measure the similarity between the generated image $G(x)$ and the target image $y_{;}$(ii) the cGAN error attempts to distinguish the generated image $G(x)$ from the target image $y$ corresponding to the input image $x$. The combined loss functions are optimized by the Adam algorithm. (B) Once trained, the generator can immediately predict the fluorescence image $y$ from the fluorescence image $x$ of the test dataset.

subjective perception, taking into account subjective factors such as brightness, contrast, structure, and resolution. However, the MS-SSIM loss function can easily lead to brightness changes and color deviations ${ }^{[16]}$. Therefore, in this work, L1 and MS-SSIM are introduced into the loss function.

The loss function of cGAN is defined as follows:

$$
\begin{aligned}
\mathcal{L}_{\mathrm{cGAN}}(G, D)= & \mathbb{E}_{x, y \sim p_{\text {data }}(x, y)}[\log D(x, y)] \\
& +\mathbb{E}_{x \sim p_{\text {data }(x)}}\{\log \{1-D[x, G(x)]\}\},
\end{aligned}
$$

where $p_{\text {data }}(x, y)$ is the joint probability distribution of the fluorescence image $x$ and the fluorescence image $y$, and $\mathbb{E}_{x, y \sim p_{\text {data }}(x, y)}$ is the expectation of log-likelihood of $(x, y)^{[18]}$. $G$ attempts to minimize the target, thereby minimizing the difference between the generated fluorescence image and the true fluorescence image, while $D$ attempts to maximize the target.

The L1 loss function can be added to ensure that the output image is clear ${ }^{[13]}$ :

$$
\mathcal{L}_{\mathrm{L} 1}(G)=\mathbb{E}_{x, y \sim p_{\text {data }}(x, y)}\left[\|y-G(x)\|_{1}\right]
$$

In addition, the MS-SSIM loss function is also added, as follows: 


$$
\mathcal{L}_{\mathrm{MS}-\operatorname{SSIM}}(G)=\mathbb{E}_{x, y \sim p_{\text {data }}(x, y)}\{1-\operatorname{MS}-\operatorname{SSIM}[y, G(x)]\} .
$$

The final loss function of our network is formulated as

$$
G^{*}=\arg \min _{G} \max _{D} \mathcal{L}_{\mathrm{cGAN}}(G, D)+\lambda_{1} \mathcal{L}_{\mathrm{L} 1}(G)+\lambda_{2} \mathcal{L}_{\mathrm{MS}-\mathrm{SSIM}}(G) .
$$

\section{Experiment and Analysis}

\subsection{Experimental dataset}

A part of the experimental dataset comes from a public dataset ${ }^{[10]}$. The data part involves three different types of cells, namely human motor neurons, human breast cancer cells, and rat cortical neurons, and eight different fluorescence images, in which Isletl protein is used as a motor neuron marker ${ }^{[19]}$, $\mathrm{TuJ} 1$ is used as a neuron marker ${ }^{[20]}$, DAPI/Hoechst is used to label cell nuclei, CellMask is used to label the cell membrane, propidium iodide (PI) is used to label dead cells ${ }^{[21]}$, MAP2 is used to label dendrites ${ }^{[22]}$, and NFH is used to label axons ${ }^{[23]}$. We randomly crop the original image into non-overlapping $256 \times 256$ images. The ratio of the training set to the test set of each group is 4:1. The detailed information of the dataset is summarized in Table 1.

In addition, the rat cardiomyocytes data from our own experiment, in which DAPI is used to label cell nuclei and CellMask is used to label cell membrane, have also been used to evaluate the generalization capability of the proposed method in Fluo-Fluo translation.

\subsection{Training}

Our deep neural network is implemented using Python version 3.8.3. cGAN is implemented using PyTorch framework version 1.6.0. Other Python libraries include torchvision, dominate,

\begin{tabular}{|c|c|c|c|c|c|}
\hline Groups & Cell Type & $\begin{array}{c}\text { Fluorescence } \\
\text { Label }\end{array}$ & $\begin{array}{l}\text { Marked } \\
\text { Location }\end{array}$ & $\begin{array}{c}\text { Training } \\
\text { Set }\end{array}$ & $\begin{array}{c}\text { Test } \\
\text { Set }\end{array}$ \\
\hline \multirow[t]{2}{*}{ Group 1} & \multirow[t]{2}{*}{$\begin{array}{c}\text { Human motor } \\
\text { neurons }\end{array}$} & Islet1 & $\begin{array}{c}\text { Motor } \\
\text { neurons }\end{array}$ & 1280 & 320 \\
\hline & & Tul1 & Neurons & & \\
\hline \multirow[t]{2}{*}{ Group 2} & \multirow{2}{*}{$\begin{array}{l}\text { Human breast } \\
\text { cancer cells }\end{array}$} & DAPI & Nuclei & 300 & 75 \\
\hline & & CellMask & Membrane & & \\
\hline \multirow[t]{2}{*}{ Group 3} & \multirow{2}{*}{$\begin{array}{l}\text { Rat cortical } \\
\text { neurons }\end{array}$} & Hoechst & Nuclei & 5760 & 1440 \\
\hline & & PI & Dead cells & & \\
\hline \multirow[t]{3}{*}{ Group 4} & \multirow{3}{*}{$\begin{array}{c}\text { Human motor } \\
\text { neurons }\end{array}$} & DAPI & Nuclei & 2400 & 600 \\
\hline & & MAP2 & Dendrites & & \\
\hline & & NFH & Axons & & \\
\hline
\end{tabular}

Table 1. Detailed Information of Experimental Data. visdom, os, time, Python Imaging Library (PIL), and Numpy. The network implementation is carried out on a desktop computer with Intel ${ }^{\oplus}$ Xeon ${ }^{\circledast}$ Gold $6248 \mathrm{R} \mathrm{CPU}$ at $3.00 \mathrm{GHz}$ and $3.00 \mathrm{GHz}$ (two processors), 256G RAM, and running Windows 10 operating system. We train the network model for 200 epochs by the Adam optimization algorithm, and the batch size of each optimization traversal is set to four. The regularization parameters $\lambda_{1}$ and $\lambda_{2}$ are both set to 100. In order to make the error converge to a smaller value, we use the learning rate attenuation strategy. The initial learning rate is set to 0.0002 . For the first 100 epochs, the same learning rate is maintained, and, for the next 100 epochs, the learning rate decays linearly to zero.

In this work, the average training time of each group is $6.8 \mathrm{~h}$, and the average test time of each image is $0.5 \mathrm{~s}$.

\subsection{Evaluation criteria}

In order to evaluate the performance of the network, we calculate the structural similarity (SSIM) ${ }^{[24]}$, the peak signal-to-noise ratio (PSNR), and the mean absolute error (MAE) between the true and the network generated images.

In this work, SSIM is calculated as

$$
\operatorname{SSIM}(x, y)=\frac{\left(2 \mu_{x} \mu_{y}+c_{1}\right)\left(2 \sigma_{x y}+c_{2}\right)}{\left(\mu_{x}^{2}+\mu_{y}^{2}+c_{1}\right)\left(\sigma_{x}^{2}+\sigma_{y}^{2}+c_{2}\right)},
$$

where $\mu_{x}$ is the average of $x, \mu_{y}$ is the average of $y, \sigma_{x}^{2}$ is the variance of $x, \sigma_{y}^{2}$ is the variance of $y$, and $\sigma_{x y}$ is the covariance of $x$ and $y \cdot c_{1}=\left(k_{1} L\right)^{2}, c_{2}=\left(k_{2} L\right)^{2}, k_{1}=0.01$, and $k_{2}=0.03$.

PSNR is defined as follows:

$$
\begin{gathered}
\text { MSE }=\frac{1}{N} \sum_{i=1}^{N}\left(x_{i}-y_{i}\right)^{2}, \\
\text { PSNR }=10 \log _{10}\left[\frac{\left(2^{n}-1\right)^{2}}{\mathrm{MSE}}\right] .
\end{gathered}
$$

MAE is calculated as follows:

$$
\text { MAE }=\frac{1}{N} \sum_{i=1}^{N}\left|x_{i}-y_{i}\right| .
$$

\section{Experimental Results and Analysis}

\subsection{Predict Fluo-Fluo translation image}

Figure 3 shows the prediction results of Groups 1-3 obtained by the proposed method. The input image, the true image, the network generated image, the absolute error map, and the scatter plot are shown, respectively. Here, the absolute error represents the difference of each pixel value between the true and the generated fluorescence images. With the higher values of absolute errors, the color of the absolute error map becomes closer to red. On the contrary, the color of the absolute error map 


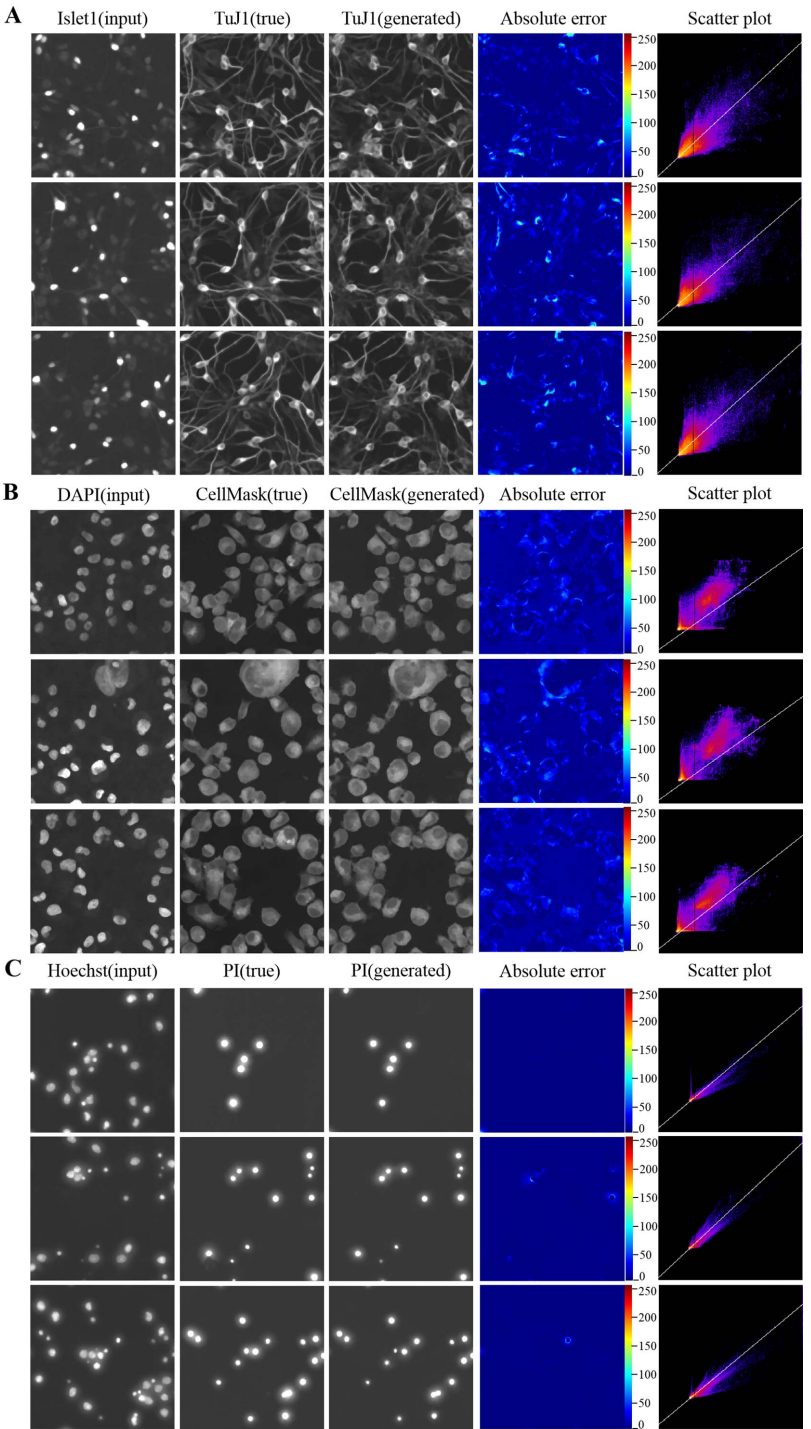

Fig. 3. Prediction results obtained by the proposed method. (A) Predict TuJ1 protein from Islet1 protein based on deep learning. (B) Predict CellMask from DAPI based on deep learning. (C) Predict PI from Hoechst based on deep learning. From left to right, the input image, the true image (ground truth), the network generated image, the absolute error map, and the scatter plot are displayed in turn.

becomes closer to blue ${ }^{[12]}$. The scatter plot is used to describe the correlation between the true and the generated fluorescence images ${ }^{[25]}$. Here, the intensity of a given pixel in the true image is used as the $x$ coordinate of the scatter plot, and the intensity of the corresponding pixel in the generated image is used as the $y$ coordinate.

\subsubsection{Predict TuJ1 protein from Islet1 protein}

The cell type is human motor neurons. Islet1 protein can be used as a motor neuron marker. TuJ1 is a tubulin involved in neuronal cell type-specific differentiation and can be used as a neuronal marker. The experimental results show that the absolute error maps [Fig. 3(a)] are mostly blue, indicating that the difference in pixel values between the true fluorescence image (true $\mathrm{TuJ} 1$ ) and the virtual fluorescence image (generated $\mathrm{TuJ} 1$ ) is very small. In addition, the distributions of the scatter plot [Fig. 3(a)] are generally in the shape of a straight bar, showing linear and diagonal distribution. That means there is a strong linear relationship between the true and the generated fluorescence images. These results indicate that cGAN can accurately predict $\mathrm{TuJ} 1$ protein from Islet1 protein.

\subsubsection{Predict CellMask from DAPI}

The cell type is human breast cancer cells. DAPI is a fluorescent dye that can bind strongly to DNA and can be used to label nuclei. CellMask dye can label membranes quickly and evenly. Based on the experimental results from Fig. 3(b), we can observe that the absolute error map is mostly blue, indicating that the pixel value difference between the true fluorescence image (true CellMask) and the virtual fluorescence image (generated CellMask) is insignificant. The scatter distribution in the scatter plot is basically linear and has a tendency to align towards the $Y$ axis. It indicates that there is a certain linear relationship between the true and the generated fluorescence images. But, the fluorescence intensity of the true fluorescence image is higher than that of the generated fluorescence image.

\subsubsection{Predict PI from Hoechst}

The cell type is rat cortical neurons. PI can enter dead cells and bind to DNA; therefore, dead cells can be identified by PI staining. As expected, the absolute error map shown in Fig. 3(c) is almost all blue, indicating that the difference in pixel values between true PI and the generated PI is small. Furthermore, the scatter distribution in the scatter plot is linear and diagonal distribution, indicating that there is a very strong linear relationship between the true and the generated fluorescence images.

\subsubsection{Predict MAP2 and NFH from DAPI}

In addition to the above prediction from a fluorescence image to another fluorescence image, we also train cGAN to predict MAP2 and NFH from DAPI and realize the prediction of a variety of other fluorescence images from one fluorescence image. Furthermore, we have achieved multi-label fluorescent staining by merging different fluorescence images generated by the network. Based on the multi-label results, we can better analyze the co-expression and co-localization of different proteins in cell samples ${ }^{[26]}$. These prediction results are shown in Fig. 4.

The cell type is human motor neurons. MAP2 is a neuronspecific cytoskeleton protein, which can be used as a neuron dendritic marker. NFH is a neuron-specific intermediate filament protein, which can be used as a neuron axon marker. The absolute error map in Fig. 4(a) is mostly blue, indicating that the pixel value difference between the true MAP2 and the generated MAP2 is minor. The distribution in the scatter plot is generally in the shape of a straight bar, indicating that there is a strong linear relationship between the true and the generated 


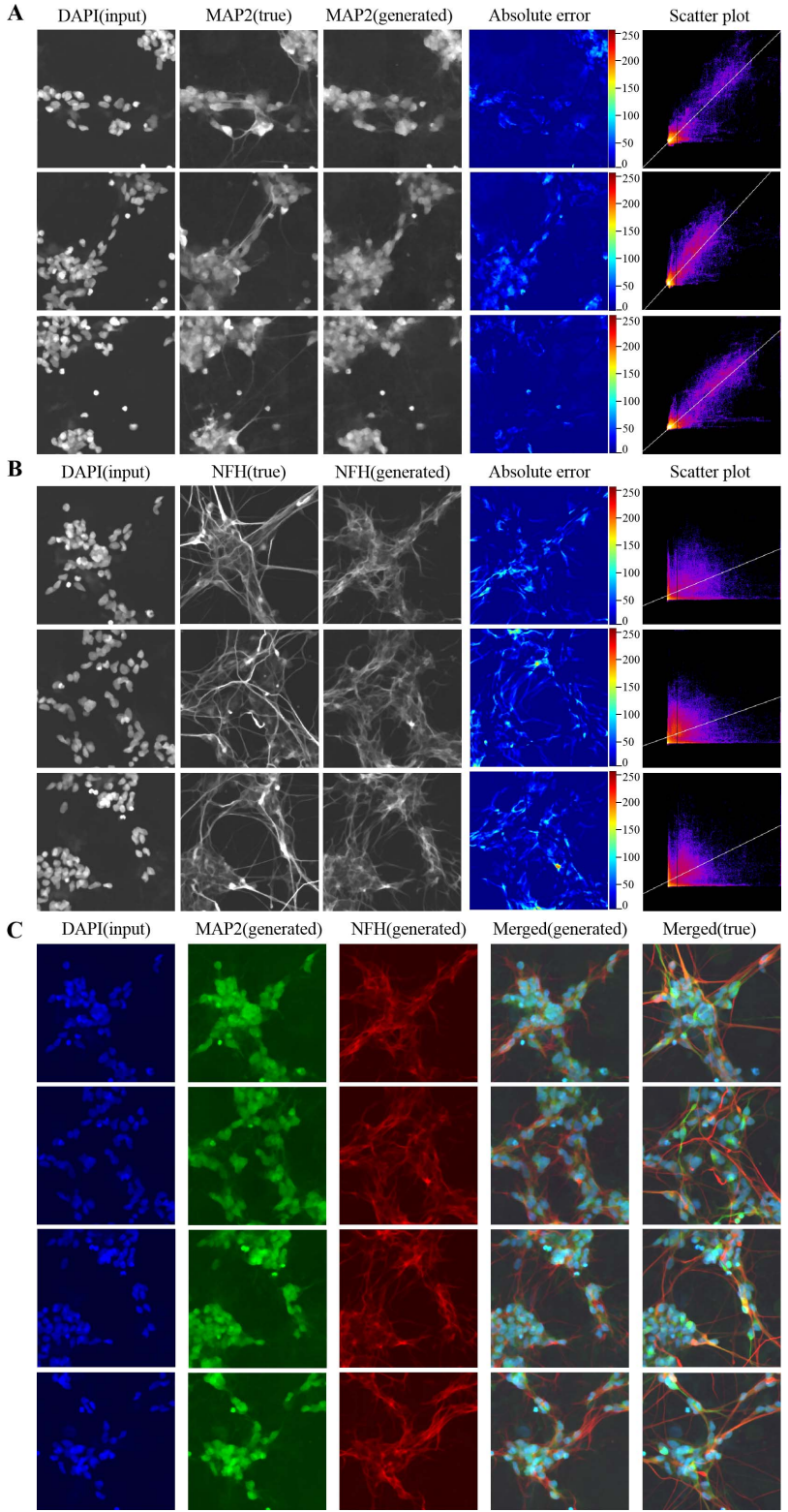

Fig. 4. Prediction results obtained by the proposed method. (A) Predict MAP2 from DAPI based on deep learning. (B) Predict NFH from DAPI based on deep learning. (C) Multi-label visualization co-localization.

fluorescence images. These results exhibit that cGAN can accurately predict MAP2 from DAPI. However, only part of the absolute error map in Fig. 4(b) is blue, indicating that there is a certain difference between the pixel values of the true fluorescence image (true NFH) and the virtual fluorescence image (generated NFH). Furthermore, the distribution of the scatter plot in Fig. 4(b) is generally fan-shaped. It means that the true and the generated fluorescence images are moderately correlated. These results demonstrate that cGAN can predict NFH from DAPI, but the prediction capability is average. Finally, Fig. 4(c) shows the merged images of various fluorescence images to visualize co-localization. The fourth column of Fig. 4(c) is the merged image of the input DAPI label and the
Table 2. SSIM, PSNR, and MAE Values Between the True and the Network Generated Images.

\begin{tabular}{|c|c|c|c|c|c|c|}
\hline \multirow[b]{2}{*}{ Comparisons } & \multicolumn{2}{|c|}{ SSIM } & \multicolumn{2}{|c|}{ PSNR [dB] } & \multicolumn{2}{|c|}{ MAE } \\
\hline & Mean & Std & Mean & Std & Mean & Std \\
\hline TUJ1 (true) vs TUJ1 (generated) & 0.802 & 0.024 & 21.845 & 0.821 & 5.682 & 1.468 \\
\hline $\begin{array}{l}\text { CellMask (true) vs CellMask } \\
\text { (generated) }\end{array}$ & 0.849 & 0.028 & 23.732 & 0.948 & 6.348 & 1.232 \\
\hline PI (true) vs PI (generated) & 0.980 & 0.009 & 29.456 & 3.266 & 0.885 & 0.610 \\
\hline $\begin{array}{l}\text { MAP2 (true) vs MAP2 } \\
\text { (generated) }\end{array}$ & 0.888 & 0.030 & 23.172 & 1.595 & 3.999 & 1.821 \\
\hline NFH (true) vs NFH (generated) & 0.729 & 0.065 & 18.130 & 1.536 & 4.616 & 1.638 \\
\hline
\end{tabular}

generated MAP2 and NFH labels. Comparably, the fifth column is the merged image of the input DAPI label and the true MAP2 and NFH labels. Compared to the above results, they are found to be similar, indicating that cGAN can effectively reconstruct a variety of fluorescence images from a single fluorescence image.

In addition to the visual comparison provided in Figs. 3 and 4, we further quantitatively compare the difference between the true and the network generated images in terms of SSIM, PSNR, and MAE. For each group, 20 randomly selected images are used to calculate the quantitative indicators. Table 2 summarizes the obtained SSIM, PSNR, and MAE values. These results further demonstrate that cGAN successfully generates virtual fluorescence images similar to ground-truth fluorescence images.

\subsection{Evaluate the effect of the number of training sets on the prediction results}

Figure 5 demonstrates the dependence of the prediction results on the number of training sets used. Figures 5(a) and 5(b) show the prediction results from Group 1 and Group 3, respectively. The outputs are obtained based on the network model trained with different numbers of training images. Furthermore, Figs. 5(c) and 5(d) quantify the differences in terms of the SSIM, PSNR, and MAE indicators. The experimental results show that with an increase in the number of training images, the prediction capability increases gradually. But, when reaching a high training data number (e.g., 2000 images in Group 3), the prediction capability seems not to be obviously improved [Fig. 5(d)]. In addition, we can also observe that relatively few training data can already produce meaningful results [Figs. 5(c) and 5(d)]. Similar phenomena have also been demonstrated in LaChance and Cohen ${ }^{[27]}$.

\subsection{Evaluate the generalization capability in Fluo-Fluo translation}

Figure 6 demonstrates the prediction capability of the proposed method by our own experimental data. The cell type of the 


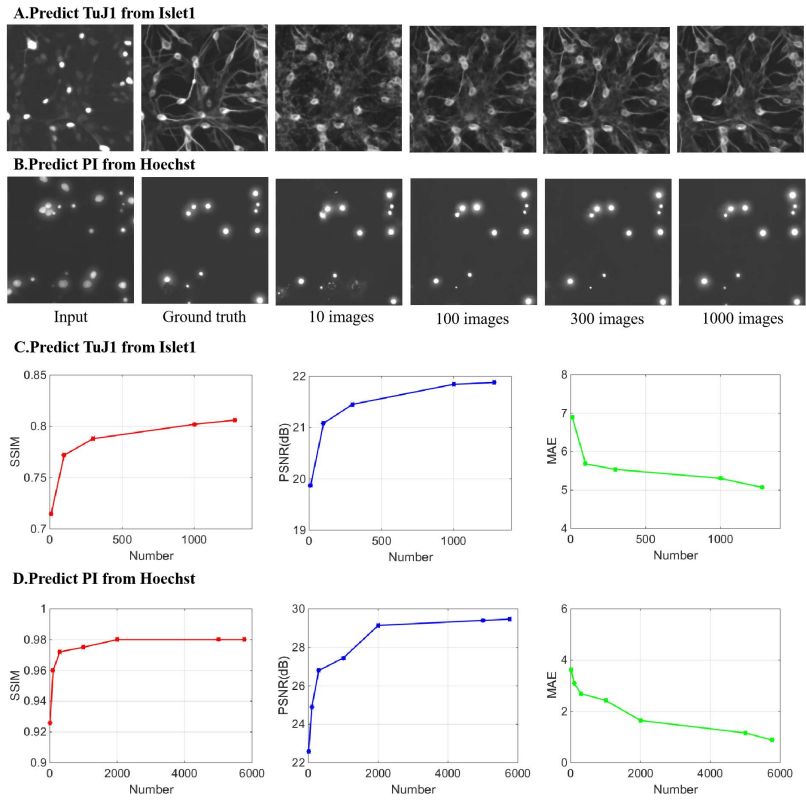

Fig. 5. Effects of the number of training sets on the prediction performance of the proposed method. (A) and (B) Prediction results obtained by using the reduced training sets. (A) Predict TuJ1 from Islet1. (B) Predict PI from Hoechst. (C) and (D) Quantify the differences in terms of the SSIM, PSNR, and MAE indicators, respectively.

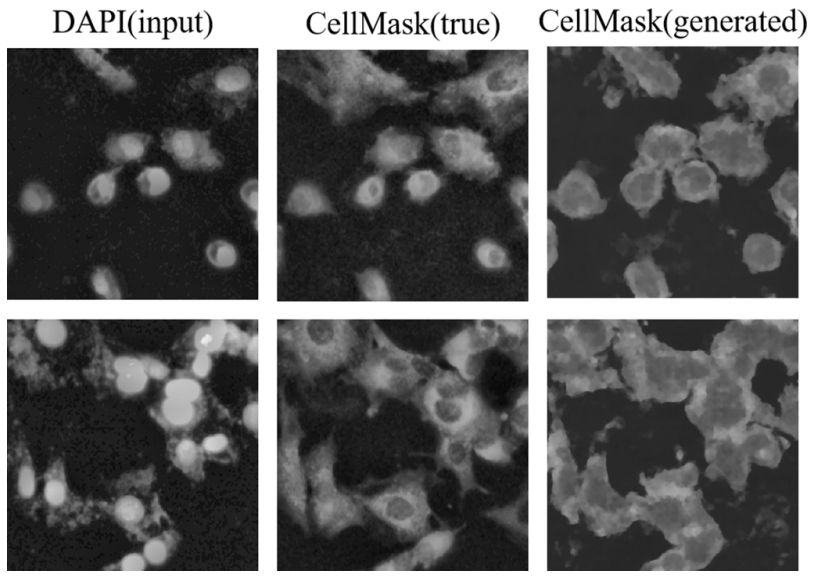

Fig. 6. Predict CellMask from DAPI based on our rat cardiomyocyte dataset. The public dataset from Group 2 (predicting CellMask from DAPI based on human breast cancer cells) is used for network training, and then the trained model is used to predict our own experimental data.

dataset is rat cardiomyocytes. DAPI is used to label the cell nuclei, and CellMask is used to label the cell membrane. To further demonstrate the generalization performance, in this case, we use the public dataset from Group 2 (predicting CellMask from DAPI based on human breast cancer cells) for network training, and then the trained model is used to predict our own experimental data. Furthermore, to calculate the quantitative indicators, 20 randomly selected test images are used. The obtained values of SSIM, PSNR, and MAE were $0.751 \pm 0.014$, $22.702 \pm 0.885 \mathrm{~dB}$, and $7.718 \pm 3.379$, respectively. These experimental results demonstrate that cGAN successfully generates virtual fluorescence images similar to true fluorescence images, even if it is applied to different datasets.

\section{Conclusion}

This work proposes a method based on deep learning to predict a fluorescence image in the same field of view from another fluorescence image. cGAN is used to generate virtual fluorescence images. To improve the quality of the generated image, we add the L1 loss function and the MS-SSIM loss function to the original loss function. In addition to intuitive visual comparison, we also use three indicators (SSIM, PSNR, and MAE) to quantitatively evaluate the performance of the network. The experimental results show that cGAN successfully generates virtual fluorescence images similar to true fluorescence images. It should be noted that we have also achieved multi-label fluorescent staining by merging fluorescent images of different labels.

In summary, this study shows that a deep neural network can achieve Fluo-Fluo translation and describe the localization relationship between subcellular structures labeled with different fluorescent markers. Our Fluo-Fluo translation method can avoid non-specific cross labeling in multi-label fluorescence staining and is free from spectral overlaps. Theoretically, an unlimited number of fluorescence images can be predicted from one fluorescence image for cell characterization.

\section{Acknowledgement}

This work was supported in part by the National Natural Science Foundation of China (Nos. 61871263, 12034005, and 11827808) and the Natural Science Foundation of Shanghai (Nos. 21ZR1405200 and 20S31901300).

\section{References}

1. A. Nwaneshiudu, C. Kuschal, F. H. Sakamoto, R. R. Anderson, K. Schwarzenberger, and R. C. Young, "Introduction to confocal microscopy," J. Investig. Dermatol. 132, e3 (2012).

2. S. Rohilla, B. Kraemer, F. Koberling, I. Gregor, and A. C. Hocke, "Multitarget immunofluorescence by separation of antibody cross-labelling via spectral-FLIM-FRET," Sci. Rep. 10, 3820 (2020).

3. P. van Horssen, M. Siebes, I. Hoefer, J. A. E. Spaan, and J. P. H. M. van den Wijngaard, "Improved detection of fluorescently labeled microspheres and vessel architecture with an imaging cryomicrotome," Med. Biol. Eng. Comput. 48, 735 (2010).

4. D. Shen, G. Wu, and H. Suk, "Deep learning in medical image analysis," Annu. Rev. Biomed. Eng. 19, 221 (2017).

5. N. Aloysius and M. Geetha, "A review on deep convolutional neural networks," in International Conference on Communication and Signal Processing (2017), p. 2.

6. E. Nehme, L. E. Weiss, T. Michaeli, and Y. Shechtman, "Deep-STORM: super-resolution single-molecule microscopy by deep learning," Optica 5, 458 (2018).

7. H. Wang, Y. Rivenson, Y. Jin, Z. Wei, R. Gao, H. Gunaydin, L. A. Bentolila, C. Kural, and A. Ozcan, "Deep learning enables cross-modality superresolution in fluorescence microscopy," Nat. Methods 16, 103 (2019). 
8. X. Chai, Q. Ba, and G. Yang, "Characterizing robustness and sensitivity of convolutional neural networks for quantitative analysis of mitochondrial morphology," Quant. Biol. 6, 344 (2018).

9. H. Shen and J. Gao, "Deep learning virtual colorful lens-free on-chip microscopy," Chin. Opt. Lett. 18, 121705 (2020).

10. E. M. Christiansen, S. J. Yang, D. M. Ando, A. Javaherian, G. Skibinski, S. Lipnick, E. Mount, A. O'Neil, K. Shah, A. K. Lee, P. Goyal, W. Fedus, R. Poplin, A. Esteva, M. Berndl, L. L. Rubin, P. Nelson, and S. Finkbeiner, "In silico labeling: predicting fluorescence images in unlabeled images," Cell 173, 792 (2018).

11. T. Nguyen, V. Bui, A. Thai, C. Raub, L. C. Chang, and G. Nehmetallah, "Virtual organelle self-coding for fluorescence imaging via adversarial learning," J. Biomed. Opt. 25, 9 (2020).

12. K. Shigene, Y. Hiasa, Y. Otake, M. Soufi, S. Janewanthanakul, T. Nishimura, Y. Sato, and S. Suetsugu, "Translation of cellular protein localization using convolutional,” Front. Cell. Dev. Biol. 9, 635231 (2021).

13. P. Isola, J. Y. Zhu, T. Zhou, and A. A. Efros, "Image-to-image translation with conditional adversarial networks," in Conference on Computer Vision and Pattern Recognition (2017), p. 2.

14. Z. Yu, Z. Ju, X. Zhang, Z. Meng, F. Yin, and K. Xu, "High-speed multimode fiber imaging system based on conditional generative adversarial network," Chin. Opt. Lett. 19, 081101 (2021).

15. J. Feng, X. Feng, J. Chen, X. Cao, X. Zhang, L. Jiao, and T. Yu, "Generative adversarial networks based on collaborative learning and attention mechanism for hyperspectral image classification," Remote Sens. 12, 1149 (2020).

16. Z. Wang, E. P. Simoncelli, and A. C. Bovik, "Multi-scale structural similarity for image quality assessment," in Asilomar Conference on Signals, Systems \& Computers (2003), p. 3.

17. S. Basavaraju, P. K. Sharma, and A. Sur, "Memorability based image to image translation," in 12th International Conference on Machine Vision (2019), p. 1.

18. D. Li, H. Hui, Y. Zhang, W. Tong, F. Tian, X. Yang, J. Liu, Y. Chen, and J. Tian, "Deep learning for virtual histological staining of bright-field microscopic images of unlabeled carotid artery tissue," Mol. Imaging Biol. 22, 1301 (2020).

19. M. Darvishi, H. G. Hamidabadi, S. S. Negah, A. Moayeri, T. Tiraihi, J. Mirnajafi-Zadeh, A. J. Jahan-Abad, and A. Shojaei, "PuraMatrix hydrogel enhances the expression of motor neuron progenitor marker and improves adhesion and proliferation of motor neuron-like cells," Iran. J. Basic. Med. Sci. 23, 4 (2020).

20. R. Matsuno, H. Ohtaki, T. Nakamachi, J. Watanabe, S. Yofu, D. Hayashi, T. Takeda, N. Nonaka, M. Seki, M. Nakamura, K. Itabashi, and S. Shioda, "Distribution and localization of pituitary adenylate cyclase-activating polypeptide-specific receptor (PAC1R) in the rostral migratory stream of the infant mouse brain," Regul. Pept. 145, 80 (2008).

21. D. Wlodkowic, W. Telford, J. Skommer, and Z. Darzynkiewicz, "Apoptosis and beyond: cytometry in studies of programmed cell death," Methods Cell Biol. 103, 55 (2011).

22. R. Kleiman, G. Banker, and O. Steward, "Development of subcellular mRNA compartmentation in hippocampal neurons in culture," J. Neurosci. 14, 1130 (1994).

23. D. C. Molliver, M. J. Radeke, S. C. Feinstein, and W. D. Snider, "Presence or absence of TrkA protein distinguishes subsets of small sensory neurons with unique cytochemical characteristics and dorsal horn projections," J. Comp. Neurol. 361, 404 (1995).

24. Z. Wang, A. C. Bovik, H. R. Sheikh, and E. P. Simoncelli, "Image quality assessment: from error visibility to structural similarity," IEEE Trans. Image Process 13, 600 (2004).

25. S. Bolte and F. P. Cordelieres, "A guided tour into subcellular colocalization analysis in light microscopy," J. Microsc. 224, 213 (2006).

26. B. G. Qian and J. Lei, "Application of multiplexed immunofluorescent staining and multi-spectral imaging in histological studies," J. Histochem. Cytochem. 26, 5013 (2017).

27. J. LaChance and D. J. Cohen, "Practical fluorescence reconstruction microscopy for large samples and low-magnification imaging," PLoS Comput. Biol. 16, e1008443 (2020). 